\title{
Study on Life Satisfaction and its Influencing Factors of Family Caregivers for Disabled People
}

\author{
Yujia Zhao \\ School of Law, Humanities and Sociology, Wuhan University of Technology, Wuhan, China
}

\begin{abstract}
The vast majority of disabled people live at home for a long time and are cared for by close relatives, while the family caregivers who cover the daily care are often under a lot of stress in terms of health, finances, relationships, etc. In this study, taking family caregivers of disabled people as the research object, more than 500 family caregivers of disabled people from six cities and counties were investigated by questionnaire, and factor analysis was used to explore the life satisfaction and its influencing factors of family caregivers of disabled people. Through the analysis, it is found that the life satisfaction of family caregivers of disabilities is generally low, and its influencing factors are ranked in order of importance: care pressure, future expectations, interpersonal communication, social support, and social integration. Therefore, China still needs to introduce more high-level social policies and take corresponding service measures from the perspective of family caregivers to effectively improve the respect and protection of the rights and interests of persons with disabilities and their families.
\end{abstract}

Keywords: The Disabled; Family Caregiver; Life Satisfaction; Influencing Factor.

\section{Presentation of Questions}

Life satisfaction usually refers to a person's subjective evaluation of the quality of life in most or a period of time in accordance with self-imposed standards [1], which is the cognitive part of subjective well-being [2]. For a long time, the research on life satisfaction in China and abroad has mainly involved three fields: quality of life, mental health and geriatrics [3]. The research on life satisfaction in Chinese academia at the present stage mostly focuses on the elderly and chronic disease groups and their caregivers. The following are the important factors that affect the life satisfaction of caregivers: the degree of disability of the elderly and the caregivers' age, education level, family monthly income, employment status, relationship with the person being cared for, health status, length of care, years of care, and burden [4]. The medical community has also carried out many studies on the life satisfaction of caregivers of various types of patients with injuries and their influencing factors. Among them, the care of stroke patients is recognized as a long-term burden for their families, which is bound to directly affect the health and life satisfaction of caregivers. While undertaking most of the nursing tasks, the attendants also bear a lot of pressure, including emotional loss, heavy responsibilities, uncertainty about the daily needs of patients, difficulty in ensuring patient safety and responsible behavior, continuous anxiety, and limited social life [5] [6]. The factors leading to low life satisfaction of patient caregivers can be summarized as concerns about continuing responsibility and constantly adding new tasks and less opportunities to pursue self-life [7] [8].

Disabled children also need comprehensive care of family caregivers. Scholars have investigated the life satisfaction of parents of disabled children, and the results show that the family caregivers of exceptional children are significantly worse than the parents of healthy children in terms of physical function [9] [10], psychological function, material life and social function, and their life satisfaction is also significantly different from the type of disability of children. For example, family caregivers of children with intellectual disabilities often have symptoms such as mental fatigue, insomnia nightmares, palpitations, chest tightness, headaches, dizziness, memory loss, and gastric ulcer. Among them, older family caregivers also suffer from chronic diseases such as rheumatism, periarthritis of shoulder, and even cataracts[11]. And because of their high levels of psychological stress, they are at greater risk for depression and mental illness, often feel anxious and tense, and exhibit persistent negative and hostile attitudes[12]. Problems such as insomnia, excessive smoking and drinking, inattention, lateness and absence from work have forced some caregivers to give up 
work and spend time with friends and relatives for entertainment, making it impossible for them to seek effective social support and resulting in declining life satisfaction[13]. The life satisfaction of parents of children with cerebral palsy was lower than that of parents of normal children, which was considered by four factors: education level, economic income, treatment duration and occupation type [14]. The life satisfaction of the primary caregivers of children with autism was also low. The great material and mental consumption, family relationship and work pressure, social discrimination, anxiety about the children's future development and negative expectations for their own retirement caused by taking care of children with autism all had an impact on their life satisfaction[15].

Reality shows that family members face great challenges in the process of caring for the disabled, which can negatively affect their health, economy, interpersonal relationship, social interaction and other aspects of life quality. However, the attention and support for family caregivers of disabled people in China is insufficient to fully cover the requirements, and relevant social policies and services need to be further implemented. At present, most relevant studies focus on the living conditions of the caregivers of disabled children and elderly disabled people. The concept that only children and elderly people need special care is accepted by default, but the impact of the care needs of elderly disabled people on the quality of their family caregivers' life is often ignored. Therefore, in this study, it is considered that the focus of research on the life satisfaction of family caregivers of disabilities should cover family caregivers of all ages and types of disabilities, and their overall life satisfaction should be comprehensively explored. In this paper, family caregivers of different ages and types of disabled persons were investigated as a whole, and the key factors affecting their life satisfaction were studied by factor analysis.

\section{Research Design}

\subsection{Research Methods}

In this study, life satisfaction of family caregivers with disabilities is measured with a self-made scale and by quantitative research method, based on the data collected from the questionnaire survey. The data for the study came from a survey on the social support status of families with disabilities. The questionnaire is structured into five parts: basic information of family members and the disabled, family economic status, family social support, psychological stress and quality of life, and family barrier-free construction. Considering the particularity of families with disabilities, the self-made scale was mainly referred to the fields considered when measuring the Quality of Life of families with disabilities in FQOLS-2006 (Family Quality of Life Survey-2006), which was designed from seven aspects of the health status, economic well-being, interpersonal communication, social tolerance, social support, leisure and entertainment and life development. As for data processing, Excel was used to input the data and SPSS 23.0 was used for analysis.

\subsection{Sample Selection}

In order to ensure the representativeness of the sample, the information used in the survey relied on the information of the disabled persons' federations of 6 cities and counties in Hubei Province. A multi-stage random sampling method was adopted, including three stages: first, towns and streets were selected by random sampling in 6 counties (cities and districts); second, natural village (neighborhood committee); third, a single sample, namely, family caregivers of disabilities. The respondents were caregivers for the disabled from six cities and counties in Hubei Province. From late August 2018 to early July 2019, a total of 550 questionnaires were distributed, and 511 valid questionnaires were obtained, with effective recovery rate of $92.91 \%$.

\subsection{Variable Selection and Assignment}

In this study, a total of 14 different variables were designed to measure the influencing factors of life satisfaction of family caregivers disabled people, including: X1: my health is affected by the care work, X2: my health will become worse, X3: after taking care of disabled members, my income fell, 
X4: my income will become worse, X5: the relationship between family members is affected, X6: relationships with friends and family is affected, X7: I often suffer discrimination from the outside world, X8: I seldom participate in community activities,X9: formal social support is effective, X10: informal social support is effective; X11: I feel I need to take a break; X12: I have been engaged in recreational activities for the past six months; X13: I am forced to abandon my original life plan; and X14: I am worried about my future.

In this study, a self-designed scale (Table 1) was mainly used to measure the life satisfaction of caregivers with disabilities. The scale consists of a group of statements, and each statement has three responses: "disagree", "general" and "agree", with scores of 1, 2 and 3. After the respondents answered all the questions, the scores of their answers were added together to obtain the total score of their life satisfaction. Because all the question items in the scale are negative statements, the higher the score is, the worse the situation of the respondent in this scale is, and the lower his life satisfaction is.

Table 1. Life Satisfaction Scale for Disabled Caregivers

\begin{tabular}{|c|c|}
\hline Field & Question Item \\
\hline Health Condition & $\begin{array}{l}\text { 1. My health is affected by the care work } \\
\text { 2. My health will become worse }\end{array}$ \\
\hline Economic Well-being & $\begin{array}{l}\text { 3. After taking care of disabled members, my income fell } \\
\text { 4. My income will become worse }\end{array}$ \\
\hline Interpersonal Relationship & $\begin{array}{l}\text { 5. My involvement in caregiving has affected my relationships with family } \\
\text { members } \\
\text { 6. My involvement in caregiving has affected my relationships with relatives } \\
\text { and friends }\end{array}$ \\
\hline Social Inclusion & $\begin{array}{l}\text { 7. I often suffer discrimination from the outside world } \\
\text { 8. I seldom participate in community activities }\end{array}$ \\
\hline Social Support & $\begin{array}{l}\text { 9. I think formal social support is less effective } \\
\text { 10. I think informal social support is less effective }\end{array}$ \\
\hline $\begin{array}{l}\text { Recreation and } \\
\text { Entertainment }\end{array}$ & $\begin{array}{l}\text { 11. I feel I need to take a break } \\
\text { 12. I seldom take part in recreational activities }\end{array}$ \\
\hline Life Development & $\begin{array}{l}\text { 13. Caregiving forced me to abandon my original life plan } \\
\text { 14. Caregiving makes me worried about my future }\end{array}$ \\
\hline
\end{tabular}

\section{Results}

\subsection{Basic Status of Samples}

The basic information of family caregivers of the disabled is as follows: in terms of gender, 189 are male, accounting for $39.38 \%$, and 289 are female, accounting for $60.21 \%$; in terms of education level, 82 have not received any education, accounting for $17.05 \% ; 138$ received primary education, accounting for $28.69 \%$; 145 received junior high education, accounting for $30.15 \%$; 94 received senior high school education, accounting for 19.54\%; and 22 received college education or above, accounting for $4.57 \%$.

The basic information of disabled people in care: in terms of age, 95 people aged 19 and below, accounting for $6.6 \%$; 100 people aged between 20 and 39, accounting for $17.5 \%$; 187 people aged between 40 and 59 , accounting for $32.6 \% ; 167$ people aged between 60 and 79 , accounting for $29.1 \%$; 
24 people aged 80 and above, accounting for $4.2 \%$; in terms of disability category, $59(11.66 \%)$ were visually disabled; 38 (7.51\%) had hearing disabilities;17 (3.36\%) had language disabilities;184 (36.36\%) were physically disabled;148 (29.25\%) were mental (intellectual) disabled;41 (8.10\%) had multiple disabilities; 15 unidentified $(2.96 \%)$; in terms of the level of disability, 82 persons had primary disabilities accounting for $16.27 \%$; 186 had secondary disabilities, accounting for 36.90\%; 99 had third-level disabilities accounting for 19.64\%; 90 had fourth-level disabilities, accounting for $17.86 \% ; 44$ have not been graded, accounting for $8.73 \%$.

\subsection{Measurement of Life Satisfaction of Disabled Family Caregivers}

In this study, life satisfaction of disabled family caregivers was analyzed as a dependent variable. Since there are many independent variables influencing it, the factors influencing the life satisfaction of disabled caregivers were summarized in the reference scale, including their own health status, economic well-being, interpersonal relationship, social inclusion, social support, leisure and entertainment, and life development, a total of 14 items. The scores of life satisfaction of the samples are shown in Table 2. Next, factor analysis is conducted on 14 projects, and several key factors affecting the life satisfaction of caregivers of disabled people are summarized.

Table 2. Scores of Life Satisfaction of Disabled Caregivers

\begin{tabular}{|c|c|c|c|c|c|}
\hline Name & Item & Disagree & Generally & Agree & Sample Score \\
\hline X1 & Health affected & 27.6 & 27.4 & 42.3 & 2.15 \\
\hline X2 & Health will be worse & 15.1 & 38.2 & 46.6 & 2.31 \\
\hline X3 & Income fell & 24.5 & 22.7 & 50.9 & 2.26 \\
\hline X4 & Incomes will get worse & 18.2 & 48.5 & 33.1 & 2.14 \\
\hline X5 & Relationships with family members are affected & 53.4 & 26.0 & 18.6 & 1.64 \\
\hline X6 & Relationships with relatives and friends are affected & 56.2 & 25.4 & 16.4 & 1.59 \\
\hline X7 & Often suffer discrimination from the outside world & 82.2 & 13.3 & 3.3 & 1.19 \\
\hline X8 & Seldom participate in community activities & 8.0 & 16.2 & 75.3 & 2.67 \\
\hline X9 & Ineffective formal social support & 24.3 & 40.1 & 35.4 & 2.11 \\
\hline X10 & Ineffective informal social support & 1.4 & 15.7 & 82.8 & 2.82 \\
\hline X11 & Feel like I need a break & 21.5 & 28.8 & 48.5 & 2.27 \\
\hline X12 & Seldom take part in recreational activities & 2.9 & 63.0 & 33.9 & 2.31 \\
\hline X13 & Forced to abandon my original life plan & 35.6 & 24.9 & 37.6 & 2.02 \\
\hline X14 & Worried about my future & 30.9 & 22.1 & 45.0 & 2.14 \\
\hline
\end{tabular}

After summing up the scores of 14 items with "agree" attitude in the sample of Table 2, the average score of the sample observation value at the level of "life satisfaction of disabled caregivers" was more than 2 , while less than 3 , specifically 2.12 , which was converted to a percentage of $70.7 \%$. That is to say, in this study, the average percentage of sample observations identified was about $70.7 \%$, indicating that the life satisfaction of disabled caregivers was generally low. When determining the high and low groups, the scores of the 14 items were first ranked in ascending order, and then the score of the $27 \%$ item was used as the criterion for classification (see Table 3 for details).

The scores in the high score group include 2.82 (X10: Ineffective informal social support), 2.67 (X8: Seldom participate in community activities), 2.31 (X2: Health will be worse), and 2.31 (X12: Seldom take part in recreational activities); accordingly, the scores in the low score group include 2.02 (X13: Forced to abandon my original life plan), 1.64 (X5: Relationships with family members are affected), 1.59 (X6: Relationships with relatives and friends are affected), and 1.19 (X7: Often suffer discrimination from the outside world).

The other items were in the middle score group, including 2.27 (X11: Feel like I need a break), 2.26 (X3: Income fell), 2.15 (X1: Health affected), 2.14 (X4: Incomes will get worse), 2.14 (X14: Worried about my future) and 2.11 (X9: Ineffective formal social support). 
X10 "Ineffective non-formal social support" is the highest score item, which shows that social support does not give effective help to disabled caregivers. Lack of recreational activities, poor health and reduced income status will also reduce the life satisfaction of disabled caregivers. Future development and life planning are also one of the most concerned issues for caregivers of disabled people.

Through the analysis of the low-level group, it can be found that the caregivers of the disabled put a lot of time and energy on the disabled, and most of them play a lot of roles at the same time, which cannot be balanced, resulting in role obstacles in interpersonal communication (X5: Relationships with family members are affected, and X6: Relationships with relatives and friends are affected). Due to social prejudices against persons with disabilities and their families, negative psychological experiences of caregivers of the disabled after being discriminated against (X7: Often suffer discrimination from the outside world) can also drag down their life satisfaction.

Table 3. Descriptive statistics

\begin{tabular}{|c|c|c|c|c|c|c|}
\hline Item & $\begin{array}{c}\text { Number of } \\
\text { Cases }\end{array}$ & Minimum & Maximum & $\begin{array}{c}\text { Sum } \\
\text { Total }\end{array}$ & $\begin{array}{c}\text { Mean } \\
\text { Value }\end{array}$ & $\begin{array}{c}\text { Standard } \\
\text { Deviation }\end{array}$ \\
\hline $\begin{array}{c}\text { X7: Often suffer discrimination from the } \\
\text { outside world }\end{array}$ & 511 & 0 & 3 & 607 & 1.19 & .493 \\
\hline $\begin{array}{c}\text { X6: Relationships with relatives and friends } \\
\text { are affected }\end{array}$ & 502 & 0 & 3 & 799 & 1.59 & .763 \\
\hline $\begin{array}{c}\text { X5: Relationships with family members are } \\
\text { affected }\end{array}$ & 502 & 0 & 3 & 824 & 1.64 & .783 \\
\hline $\begin{array}{c}\text { X13: Forced to abandon my original life } \\
\text { plan }\end{array}$ & 502 & 0 & 3 & 1012 & 2.02 & .868 \\
\hline $\begin{array}{c}\text { X9: Ineffective formal social support } \\
\text { X14: Worried about my future }\end{array}$ & 510 & 1 & 3 & 1077 & 2.11 & .766 \\
\hline X4: Incomes will get worse & 511 & 0 & 3 & 1096 & 2.14 & .708 \\
\hline X1: Health affected & 498 & 0 & 3 & 1069 & 2.15 & .840 \\
\hline $\begin{array}{c}\text { X3: Income fell } \\
\text { X11: Feel like I need a break }\end{array}$ & 502 & 0 & 3 & 1137 & 2.26 & .840 \\
\hline X12: Seldom take part in recreational & 506 & 0 & 3 & 1148 & 2.27 & .803 \\
\hline activities & 511 & 0 & 3 & 1178 & 2.31 & .532 \\
\hline X2: Health will be worse & 511 & 0 & 3 & 1181 & 2.31 & .727 \\
\hline X10: Ineffective informal social support & 510 & 1 & 3 & 1436 & 2.82 & .422 \\
\hline Number of valid cases (in columns) & 495 & & & & & .628 \\
\hline
\end{tabular}

\subsection{Exploratory Factor Analysis on Life Satisfaction of Caregivers of Disabled People}

(1) Reliability and validity analysis of the questionnaire

Reliability refers to the reliability of the survey results, which is generally greater than 0 and less than 1 . If the reliability coefficient exceeds 0.6 and is closer to 1 , it indicates that the reliability is higher. After each item was recoded, the total scores of the 14 questionnaire items were calculated. The statistical test results showed that all the 14 items tested reached the significance level $(\mathrm{P}<0.01)$. The details of the Alpha reliability coefficient analysis of the sample data are shown in Table 4. Cronbach's Alpha obtained is 0.744 , which means that all items have good internal consistency and strong discrimination ability, and can be analyzed in the next step. Validity mainly involves two parts, one of which is structural validity, and the other is content validity that focuses on measuring the true degree of the results. If the KMO value exceeds 0.6 , factor analysis can be conducted. The KMO test 
value and Bartlett test value obtained after the $\mathrm{KMO}$ and Bartlett tests of the questionnaire (see Table 5) are 0.755 and $1786.006(\mathrm{DF}=91, \mathrm{P}=0.000)$, the correlation matrix of data is not the identity matrix and can be used for factor analysis.

Table 4. Reliability Statistics of Cronbach's $\alpha$ Coefficient Test

\begin{tabular}{|c|c|c|}
\hline Cronbach's $\alpha$ & Cronbach's $\alpha$ Based on Standardized Term & Number of Terms \\
\hline .744 & .719 & 14 \\
\hline
\end{tabular}

Table 5. KMO and Bartlett Sphericity Test

\begin{tabular}{|l|c|c|}
\hline \multicolumn{2}{|c|}{ KMO Measure of Sampling Adequacy } & .755 \\
\hline \multirow{3}{*}{ Bartlett Sphericity Test } & Approximate Chi-square & 1786.006 \\
\cline { 2 - 3 } & Degree of Freedom & 91 \\
\cline { 2 - 3 } & Significance & .000 \\
\hline
\end{tabular}

(2) Factor Analysis

In the process of data analysis, principal component analysis was adopted, and the results showed that there were 5 factors with characteristic roots greater than 1 . Then by observing the scree plot, it was found that the data became more and more frequent after the beginning of the fifth factor, so the five common factors were determined and proposed, and the cumulative variance contribution rate reached $64.954 \%$, indicating that these five factors could explain $64.954 \%$ of the total variation. According to sociological research, if the cumulative variance contribution rate exceeds $50 \%$, the final result is acceptable. Orthogonal rotation of the maximum variance method was carried out for 14 items. After the variance-maximized rotation was converged for 7 iterations and the coefficient less than 0.4 was removed, the sorted rotation component matrix was obtained (see Table 6).

Table 6. Factor Rotation Matrix

\begin{tabular}{|c|c|c|c|c|c|}
\hline \multirow{2}{*}{ Item } & \multicolumn{5}{|c|}{ Component } \\
\hline & F1 & F2 & F3 & F4 & F5 \\
\hline X1: Health affected & .750 & & & & \\
\hline X3: Income fell & .704 & & & & \\
\hline X11: Feel like I need a break & .729 & & & & \\
\hline X13: Forced to abandon my original life plan & .691 & & & & \\
\hline X14: Worried about my future & .757 & & & & \\
\hline X2: Health will be worse & & .875 & & & \\
\hline X4: Incomes will get worse & & .863 & & & \\
\hline X5: Relationships with family members are affected & & & .779 & & \\
\hline X6: Relationships with relatives and friends are affected & & & .781 & & \\
\hline X7: Often suffer discrimination from the outside world & & & .640 & & \\
\hline X9: Ineffective formal social support & & & & .804 & \\
\hline X10: Ineffective informal social support & & & & .696 & \\
\hline X12: Seldom take part in recreational activities & & & & & .831 \\
\hline X8: Seldom participate in community activities & & & & & .643 \\
\hline
\end{tabular}


(3) Factor analysis results

Five factors (including 14 items ) were extracted from the exploratory factor analysis, indicating that the factors affecting the life satisfaction of caregivers of persons with disabilities involved a total of five aspects, namely, care stress factors, future expectations factors, interpersonal factors, social support factors and social integration factors. Their importance was ranked from high to low, as follows :

Factor 1 includes five items, X1, X3, X11, X13 and X14, which mainly reflects that the current care work brings great burden to all aspects of the life of the caregivers of the disabled, thus putting pressure on the caregivers of the disabled, so it is named as "care pressure factor".

Factor 2 includes X2 and X4 items, which mainly reflect the tendency of caregivers of disabled people predicted that their health and income had a trend towards negative changes in the future, so it is named "future expectation factors".

Factor 3 includes X5, X6, and X7, which mainly reflect that the care work changes the relationship between caregivers with disabled persons and family members and relatives and friends, and they also suffer discrimination from relatives and friends, community neighbors, colleagues, service personnel, government staff, and strangers, which further increases their barriers in interpersonal communication, hence the name "interpersonal factor".

Factor 4 includes X9 and X10 items, which mainly reflect the current situation that caregivers for the disabled receive unsatisfactory formal and informal social support and are unable to spare time and energy for leisure activities based on the lack of external help, so it is named "social support factors".

Factor 5 includes X8 and X12, which mainly reflects the low frequency of leisure and entertainment and community participation of caregivers for the disabled, indicating that they have fewer opportunities to contact and integrate into the outside world, so it is called "social inclusion factor".

\section{Discussion on Influencing Factors of Quality of Life for Family Caregivers of Disabled People}

After further dimension reduction analysis of all the data, five major factors affecting life satisfaction of caregivers for the disabled were found to be care stress, future expectation, interpersonal interaction, social support, and social integration.

\subsection{Stress Factors in Care}

Caregiver stress is an important factor influencing life satisfaction of caregivers for the disabled. Due to disability, disease, old age and other reasons, people with care needs have lost their self-care ability to varying degrees, and their daily life must be assisted by others or completely dependent on others. In view of the specific national conditions of the community nursing is still in its infancy, along with the traditional family concept, the care for the disabled in China is mainly realized through family help. The close relatives of the disabled, as the most important caregivers, make up for the lack of medical care resources to a certain extent, but at the same time they also bear a heavy burden of care [16].

Health and rest status will affect the life satisfaction of family caregivers of disabled people. Most scholars believe that care burden has a negative impact on caregivers' life satisfaction [17]. In the survey, $75 \%$ of respondents said they really wanted to take a break, indicating that caregivers of disabled people are looking for a respite from stress and exhaustion. The daily workload of family caregivers for the disabled is very heavy and tedious. As time passes, their physical and mental health deteriorates with the increase of their physical load, which is bound to lower their life satisfaction.

Income also affects the life satisfaction of disabled family caregivers. Close-fitting care makes it necessary for caregivers of persons with disabilities to adjust their working hours and even quit their work in order to better assume the role and tasks of caregivers, which directly leads to a decrease in 
their income. The increase of economic burden is not only likely to deteriorate the quality of life of disabled caregivers, but also indirectly affect their level of happiness by changing their health status and psychological pressure.

Life development planning will affect the life satisfaction of disabled family caregivers. Longterm care work makes most caregivers unable to balance the relationship between family care and individual employment. Many people have to give up their original life planning and spare a lot of time and energy to focus on care because of their consideration to the reality of family relations, maintenance obligations and family economic level. Bounded in a limited living environment, caregivers of the disabled not only have to sacrifice their personal lives but also face difficulties in the care process. This kind of end point of care life with a sense of incompetence is far away, which will inevitably lead to anxiety and worry about the future development of the disabled caregivers.

\subsection{Future Expectation Factors}

Future expectations are an important factor affecting the life satisfaction of most disabled caregivers.

Future expectations come from the dual cognition and evaluation of self-identity and social identity of social members. Positive expectation can improve residents' happiness, while negative expectation and social anxiety can reduce people's self-identity and social identity, leading to the lack of happiness [18]. In this study, the impact of future expectations of caregivers of disabled people on their life satisfaction is mainly reflected in health expectations and income expectations.

The expectation of poor physical quality will affect the life satisfaction of family caregivers of disabled people. From the perspective of psychological mechanism, suggestion is a kind of assumption that is affirmed by subjective intention. Although it may not have practical basis, it tends to this content psychologically because it has been affirmed subjectively [19]. Therefore, the negative psychological suggestion will further bring negative perceptions and experience to the caregivers of the disabled, imperceptibly make them reduce their adaptive capacity to stress, underestimate their health level, especially go against improving their mental health level, thus affecting their current life satisfaction.

The expectation of poorer income will affect the life satisfaction of family caregivers with disabilities. For caregivers of disabled people, in addition to meeting the daily needs of other family members, the medical rehabilitation, education and employment of disabled members will be a considerable continuous cost. Empirical research shows that positive income expectations have a significant role in promoting the public's subjective well-being [20], whereas the declining trend of income will undermine the confidence and courage of caregivers with disabilities when anticipating future economic difficulties, which is not helpful to stabilize one's mood and dealing with current work challenges.

\subsection{Interpersonal Communication Factors}

Interpersonal communication will affect the life satisfaction of family caregivers of disabled people.

Individual satisfaction with relationships with family members and friends can predict all dimensions of subjective well-being [21]. Meanwhile, the decrease of social contact will influence the happiness experience of caregivers with disabilities to some extent.

The change of relationship with relatives and friends will significantly affect the life satisfaction of family caregivers of disabled people. When the disabled caregivers encounter trouble, they choose to tell their relatives and friends who are more familiar with them. However, long-term care work makes them feel tired, nervous, accompanied by anxiety, depression and other negative emotions, affecting the state of living with others; some interviewees feel it is not convenient to talk too much about family matters with outsiders, so they are reluctant to express their true feelings too much. At the same time, the inability or inability of some relatives and friends to provide effective help will reduce the trust of disabled caregivers to them, resulting in interpersonal psychological distance 
between each other, which increases the loneliness of disabled caregivers, and then decreases the quality of life and satisfaction [22].

Changes in the relationship with family members will affect the life satisfaction of family caregivers of disabled people. According to role theory, the poor relationship between caregivers of persons with disabilities and family members is mainly due to role conflicts that may arise when they play different roles within the family. For most people with disabilities, their primary caregivers are their children, parents, daughters-in-law or spouse. When these people need to play the role of caregiver for the disabled in addition to taking on the specific role of relatives and fulfilling corresponding obligations, conflicts and coordination between them are easy to occur. Family conflicts caused by subjective reasons such as inadequate capacity and recognition of the role of caregivers with disabilities, and objective reasons such as different actors' different expectations of the role bearers, deviation of role distance and differences between different roles, will inevitably affect their relationship with family members [23].

The degree of social inclusion will also affect the life satisfaction of family caregivers of disabled people, and it is one of the important factors that determine whether they can smoothly carry out interpersonal communication. The misunderstanding, discrimination and prejudice of the public towards the disabled will spread to the caregivers of the disabled, causing them to bear the psychological pressure of shame and loneliness, thus reducing the possibility for them to actively seek opportunities for communication and expand the scope of communication. Studies have shown that loneliness and social exclusion significantly reduce residents' life satisfaction. The more disabled family caregivers feel lonely, the more discrimination and unfair treatment they receive, the lower their life satisfaction [24].

\subsection{Social Support Factors}

Social support will have an important impact on the life satisfaction of family caregivers disabled people. Social support is a social behavior or activity accompanied by social vulnerable groups, which can be divided into formal support and informal support. If life satisfaction represents an individual's cognitive evaluation of positive emotions based on cognitive information, there is a significant positive correlation between different forms of social support and overall social support and life satisfaction, that is, the more social support an individual gets, the stronger his subjective well-being will be. In order of their influence, emotional support, instrumental support and information support have significant predictive effects on subjective well-being [25].

The effect of informal social support will affect the life satisfaction of family caregivers of disabled people. Scores show that family caregivers for persons with disabilities have a high recognition rate of "poor effectiveness of informal social support". Informal social support is the main source of emotional support and information support, which exists in social relationships such as friends and relatives, and significantly affects health-related quality of life dimensions. Such informal support can not only improve the physical and mental health of caregivers of persons with disabilities, but also enable individuals to enjoy development experiences, such as increasing social confidence, setting life goals, achieving life values and promoting self-development.

The effect of formal social support will also affect the life satisfaction of family caregivers of disabled people. Compared with the effect of "poor informal social support" on the life satisfaction of disabled caregivers, the effect of "poor formal social support" on the life satisfaction of disabled caregivers is slightly weaker, partly because the total amount of formal social support received by them as a whole is insufficient. At present, the existing formal social support for disabled people mainly for tools and information support, i.e., to provide them with certain economic aid, material support, or specific advice guidance, etc., but there is insufficient concern to their family caretakers, and when their needs are not met, their enthusiasm and professionalism in caring can be seriously affected. 


\subsection{Social Integration Factors}

Social integration is the influencing factor of life satisfaction of family caregivers of disabled people. From the perspective of sociology, social integration is expressed as "a process in which vulnerable subjects actively engage in reflective and continuous interaction with individuals and groups in specific communities [26]". Family caregivers of disabled people face greater difficulties in participating in recreational activities, community activities and social integration, resulting in lower life satisfaction.

The frequency of recreational activities affects the life satisfaction of family caregivers of disabled people. From the quality-of-life assessment survey carried out by the World Health Organization, it is found that the factor of "leisure and recreation participation opportunities" in the field of "social environment" contributes greatly to the improvement of quality of life and is a key factor affecting quality of life [27]. The survey shows that most of the disabled family caregivers' daily leisure is dominated by eating, sleeping and watching TV. However, such time-consuming leisure activities have no significant effect on improving life satisfaction, and tourism, reading and learning are positive leisure activities that can have a significant positive impact on life satisfaction. Therefore, even if the caregivers of the disabled have the opportunity to rest and entertain in family life, their leisure activities are not high in quality due to the constraints of time constraints and low economic level, which cannot significantly improve their life.

The frequency of participation in community activities will affect the life satisfaction of family caregivers of disabled people. A comparative study of happiness in 41 countries shows that community participation and social activities are also closely related to happiness [28]. The caregivers of the disabled have low community participation and the lack of sense of community makes them more humble and sensitive, unable to feel their own existence value and difficult to harvest social identity, which is also one of the reasons for their low life satisfaction.

\section{Conclusion}

The life satisfaction of family caregivers of disabled people is generally low, and the factors affecting their life satisfaction involve five aspects, namely, care pressure, future expectation, interpersonal communication, social support and social integration. Specifically, most of the care pressure of the disabled family caregivers comes from personal health, lack of rest, poor income, and poor life development planning; poor future expectations mainly include negative estimates of the trend of deterioration of physical fitness and income reduction; the frustration of interpersonal experience is mainly reflected in the changes in the relationship between family caregivers and relatives and family members; the insignificant effect of social support is mainly due to the poor effect of informal social support for disabled people and their families and the insufficient total amount of formal social support; the low degree of social integration can be attributed to the low frequency of high-quality leisure entertainment and participation in community activities by family caregivers of disabled people.

China still needs to introduce more high-level social policies and service measures from the perspective of family caregivers to effectively improve the respect and protection of the rights and interests of persons with disabilities and their families. Protecting the quality of material life of family caregivers of disabled people from the aspects of health and health, economic and occupational development helps to increase their opportunities to enjoy recreational activities and develop spiritual civilization. Greater efforts should be made in medical assistance, poverty alleviation, two subsidies, subsistence allowances and educational assistance to alleviate the real burden and concerns of families with disabilities and to enhance their confidence and aspirations for future life. It is necessary to strongly support community organizations represented by disabled federations and grass-roots organizations represented by neighborhood ( village ) committees to directly face families with disabilities and provide them with more sympathy and psychological counseling. The ethos of "equality, participation and sharing" should be advocated by the whole society, barrier-free 
environment construction should be promoted, and discrimination and prejudice against the disabled should be eliminated in order to help family caretakers of the disabled to further participate in society.

\section{References}

[1] Ed Diener. Traits Can Be Powerful, but Are Not Enough: Lessons from Subjective Well-Being [J]. Journal of Research in Personality, 1996, 30(3) : 389-399.

[2] D. C. Shin and D. M. Johnson. Avowed Happiness as an Overall Assessment of the Quality of Life [J]. Social Indicators Research, 1978, 5(1-4) : 475-492.

[3] Wang Wei, Yang Junsheng, Xin Zhiyong. Research Results and Its Significance and Value on Life Satisfaction Abroad [J]. Journal of Jiangxi Normal University (philosophy and social science edition), 2010, 43(04):42-45.

[4] Hou Weiwei, Wang Yuhuan, Feng Yanan. Correlation between Life Satisfaction and Care Burden of Informal Caregivers at Home [J]. Chinese Journal of Gerontology,2014,33(19):4806-4810.

[5] Bakas Tamilyn et al. Time and Difficulty of Tasks Provided by Family Caregivers of Stroke Survivors.[J]. The Journal of neuroscience nursing: journal of the American Association of Neuroscience Nurses, 2004, 36 (2): 95-106.

[6] Coombs Ursula Eileen. Spousal Caregiving for Stroke Survivors.[J]. The Journal of neuroscience nursing : journal of the American Association of Neuroscience Nurses, 2007, 39(2) : 112-9.

[7] Forsberg-Wärleby Gunilla and Möller Anders and Blomstrand Christian. Spouses of First-ever Stroke Victims: Sense of Coherence in the First Phase after Stroke.[J]. Journal of rehabilitation medicine, 2002, 34 (3): 128-33.

[8] A.P. Nieboer et al. Spousal Caregivers' Activity Restriction and Depression: A Model for Changes over Time [J]. Social Science \& Medicine, 1998, 47(9) : 1361-1371.

[9] Zhou Changhong. Study on the Correlation between Quality of Life and Coping Styles of Parents of Autistic Children [J]. Chinese Journal of Behavioral Medicine and Brain Science,2010 (12):1113-1115.

[10] Ma Xinrong. Correlational Research on the Quality of Life and Sleep of Parents of Autistic Children [J]. Sichuan Mental Health,2011(2):77-79.

[11] Tao Rui. Analysis of Policy Support for Family Caregivers --A Case Study of Family Caregivers of Cerebral Palsy Children in Guangzhou [J]. Chinese Public Administration,2013(8):49-52.

[12] Liu Lamei, Zhou Lanshu, Lv Weibo, Peng Peng. Analysis of Research Status of Family Caregivers in China [J]. Nursing Journal of Chinese People's Liberation Army,2007(09):51-53.

[13] Li Fangfang, Yang Liu. Research Progress on Social Support for Family Caregivers of Special Shildren in China in Recent Ten Years [J]. Modern Special Education,2015(08):9-12+80.

[14] Li Runjie, Yang Ying, Cao Chunjing. Analysis of Life Satisfaction and Related Factors of Parents of Children with Cerebral Palsy [A]. Child Rehabilitation Professional Committee of Chinese Rehabilitation Association, Rehabilitation Committee of Pediatric Cerebral palsy, China Association for Rehabilitation of Disabled Persons, Proceedings of the 4th National Children's Rehabilitation, the 11th National Children's Cerebral Palsy Rehabilitation Academic Conference and international Academic Exchange Conference[C], Child Rehabilitation Professional Committee of Chinese Rehabilitation Association, Rehabilitation Committee of Pediatric Cerebral Palsy, China Association for Rehabilitation of Disabled Persons:Chinese Association of Rehabilitation Medicine,2010:2.

[15] Chen Chen. Research on Group Work Intervention of Mental Stress of Caregivers for People with Intellectual Disabilities [D]. East China University of Science and Technology,2013.

[16] Tang Juanjuan, Wang Junjie, Wang Yongjian. Study on the Correlation between the Nursing Burden and Quality of Life of Family Caregivers and the Degree of Disability of the Elderly [J]. Nursing Research, 2016, 30 (20):2444-2448.

[17] Hou Weiwei, Wang Yuhuan, Feng Yanan. Correlation between Life Satisfaction and Care Burden of Informal Caregivers at Home [J]. Chinese Journal of Gerontology,2014,33(19):4806-4810.

[18] Li Lei, Liu Bin. Influence of Expectation on Subjective Well-being of Urban Residents in China [J]. Nankai Economic Studies,2012 (4) :53-67. 
[19] Liang Yong, Zhang Ning, Zou Mengxuan, Han Yangyang, Pan Yueda, Ye Xinyu. Analysis of Influencing Factors of Health Status of Chinese Adults Based on Health Demand Model [J]. Medicine and Society, 2015, 28(07):50-53.

[20] Zhao Xinyu, Fan Xin, Jiang Yang. Income, Expectation and Subjective Well-being: An Empirical Study Based on Chinese Questionnaire Data [J]. Economist,2013(09):15-23.

[21] Kee-Lee Chou. Social Support and Subjective Well-Being Among Hong Kong Chinese Young Adults[J]. The Journal of Genetic Psychology, 1999, 160(3) : 319-331.

[22] Cao Xiaojun, Dai Li. Relationship between Interpersonal Communication Ability and Life Satisfaction of College Students [J]. China Journal of Health Psychology,2020,28(06):914-918.

[23] Zhang Min, Li Xiao. Research on the Role Conflict of Family Caregivers of Rural Disabled Elderly [J]. Journal of Yunnan Agricultural University (social sciences),2020,14(04):46-53.

[24] Yao Yelin. Life Satisfaction of Chinese Urban Residents from the Perspective of Social Quality --A Case Study of Six Capital Cities in China [J]. Journal of Jinan University (social science edition), 2020,30 (04): 146-145+160.

[25] Xing Zhanjun, Zhang Yu. Research on the Relationship between Social Support and Subjective Wellbeing [J]. Social Science Research, 2007 (06) : 9-14.

[26] Zhang Wei, Xu Zhe. Strategies of Social Integration and Social Work Intervention of Urban and Rural Migrant Families from the Perspective of Social Support [J].Chinese Social Work, 2020 (28) : 32-33.

[27] Lai Xiaowei. Impact of Community Leisure Participation on Improving the Quality of Life of Special Groups --A Case Study Based on Foreign Countries [J]. Journal of Hubei Institute of Technology (humanities and social sciences edition), 2017,34 (05) : 19-24.

[28] Zhu Wenjuan. Health, Security and Social Integration: A Study on Subjective Well-being of the Elderly from the Perspective of Positive Aging [J]. Journal of Hunan University of Finance and Economics, 2020, $36(03): 38-47$. 